\title{
To Fix Or Not To Fix.
}

\author{
Elaine Humphrey
}

\section{University of Victoria, Victoria, British Columbia, Canada}

From the beginning of electron microscopy, biologists learned that putting a water-loaded cell structure in an electron microscope is fraught with artifacts as the vacuum sucks the water out of the cell and alters its configuration. The better the vacuum the better the resolution of the microscope and the faster the destruction occurs.

However, any kind of fixation of the cell to preserve its structure is a compromise. Once a protocol seems to work, we frequently say "if it ain't broke, don't fix it" and subsequent images are compared to the original protocol. That is until some bright spark comes up with a better way of doing things. Fortunately, this happens especially as new instrumentation is developed. Thirty years ago say, the thought of looking at parts of a bacterium, never mind a virus was a wonderful dream. Now, even in my lab, we have microscopes that can look at atoms.

But Biologists still wanted to look at living cells and follow metabolic processes. Since you cannot do that with a typical electron microscope, fluorescent and confocal systems were developed. However, if you hit a mammalian cell with short wavelengths such as blue light, they will die. Some may survive days but more often 20-30 minutes. Antifade substances were important to keep the fluorescence working, all compromising the condition of the cells. Fixing the cells at different stages of the metabolic process gave insights but following the process in real time was the goal and techniques to keep the cells alive were still a compromise. Then multiphotons with longer and less damaging wavelengths made their appearance and later super resolution microscopes were developed.

It turns out that many insects have a shielding (outside chitin skeleton) that allows time to obtain images in the live state in a scanning electron microscope. Wood ticks aren't phased by the beam or the vacuum and even move about in the vacuum (Figure 1).

Variable pressure electron microscope systems and wet cells chambers mean you can use an electron microscope to look at live material at electron microscopy level, for a short time at least and with limited resolution.

As time went by, improvements in processing samples happened while improvements in instrument resolutions happened.

Much has been made about what constitutes a correct image of the structures in a living cell and how much compromise can be allowed. Comparing a glutaraldehyde fixed cell against a high-pressure frozen cell provided much debate as to which was correct. Now at least we can agree that cryo-fixation provides the most like-life structures. So why are labs still using aldehyde fixtion: particularly with microwave processing? Cost and speed. Being able to process a sample in about two hours rather than two days, means a researcher can get answers very fast at a reasonable cost (Figure 2).

We experimented with comparing conventional aldehyde fixation with that of using a microwave, and cryo fixation using a high-pressure freezer of animal and plant tissue. Formaldehyde is a single fixative, which means when you put the samples in buffer for storage, the fixation will reverse. Always store with 
diluted formaldehyde. Glutaraldehyde is a double fixative and leaving the samples in the fix for a long time shows over-fixation. Always store in buffer. Glutaraldehyde is slower to penetrate tissues than formaldehyde. A mixture of them both generally gives better fixation.

In general then, for electron microscopy, it is not a question of "to fix or not to fix" it is more of a question "how can I process this sample to get the best representation of the living cell that I can" or "what fixation method do I use?" together with "what microscope do I use?"

To fix or not to fix depends on the specimen, the investigation being performed, and the instruments used.
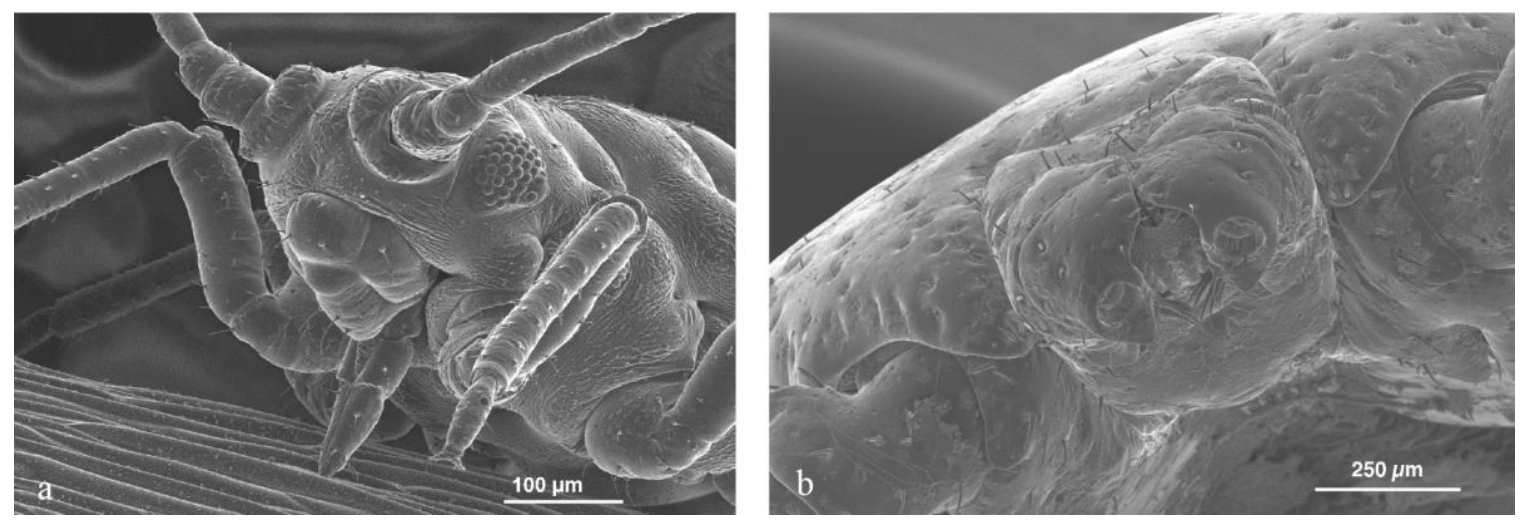

Figure 1. Not fixed: live specimens in a Hitachi S2600 variable pressure sem. 1a Aphid feeding on Arapidopsis 1b. Woodtick
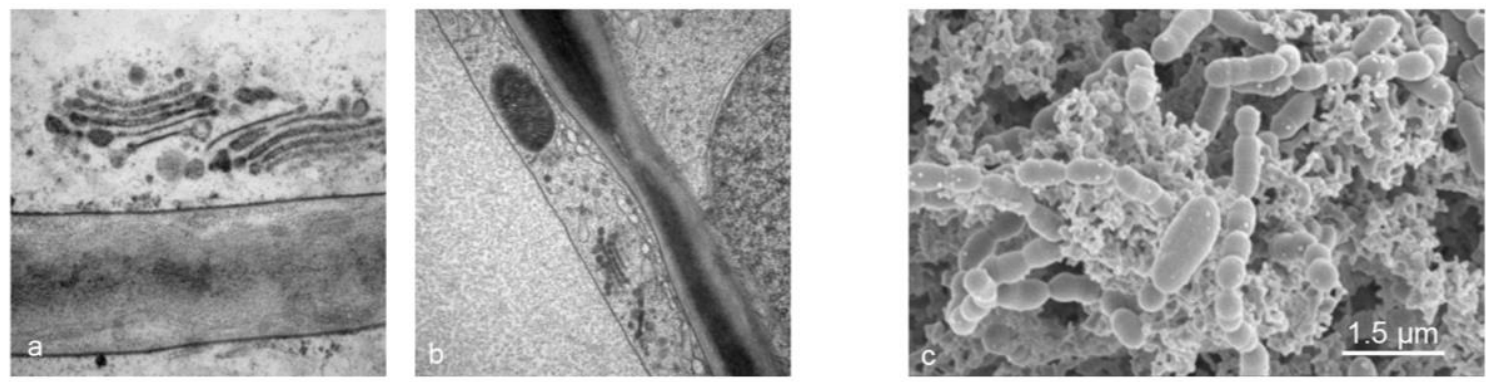

Figure 2. Fixed examples $2 \mathrm{a}$ and $2 \mathrm{~b}$ Arabidopsis thaliana leaf and stem samples were high pressure frozen, automatically freeze substituted at $-850 \mathrm{C}$ and brought up to room temperature. Resin infiltration conventionally vs microwave. Viewed in a Hitachi H7600 TEM. 2a conventional resin infiltration 2b microwave infiltration 2c. plump bacterial cells microwave processed, HMDS dried.

\section{References}

The work of this paper is accumulated knowledge from working in multi user, multi-instrument, multidisciplinary labs. 